\title{
Characterization of Etched and Grown GaN-GaN Schottky Diodes
}

\author{
Prudhvi Peri $^{1 *}$, Kai Fu ${ }^{2}$, Yuji Zhao ${ }^{2}$, David J. Smith ${ }^{3}$
}

1. School for Engineering of Matter, Transport and Energy, Arizona State University, Tempe, AZ 85287, USA

2. School of Electrical, Energy and Computer Engineering, Arizona State University, Tempe, AZ Electrical

3. Department of Physics, Arizona State University, Tempe, AZ

*Contact: pperi1@asu.edu

GaN-based devices are of much current interest for high-power electronics due to their superior physical and electrical properties, which include high electric breakdown field, high operation temperature, large band gap, and high electron velocity. GaN devices grown on $\mathrm{GaN}$ substrates have greatly reduced dislocation density $\left(<10^{6} \mathrm{~cm}^{-2}\right)$ compared to heteroepitaxial GaN $\left(10^{8}-10^{10} \mathrm{~cm}^{-2}\right)$, which should assist in improving device performance for high-power applications as well as providing increased energy conversion efficiency [1]. The commercialization of GaN-based devices is also possible due to the wide availability of freestanding GaN substrates grown by hydride vapor phase epitaxy. In this study, GaNon-GaN Schottky diodes have been examined for the presence of dislocations as well as any damage caused in $p$-GaN layers grown after chemical etching. Samples were grown homo-epitaxially on 2-inch $c$-plane $n+\mathrm{GaN}$ substrates using metal-organic chemical vapor deposition [2]. Nine microns of unintentionally doped (UID) GaN was then grown on the GaN substrates for all samples. A reference sample labeled as NE was then grown with an additional 25nm UID GaN layer, whereas samples labeled E25 and E58 were etched using low-power inductively-coupled plasma (ICP) followed by growth of UID GaN with thicknesses of $25 \mathrm{~nm}$ and $58 \mathrm{~nm}$, respectively. 500-nm of Mg-doped $p$-GaN was then deposited on all three samples. The doping concentrations in the $p$-GaN layers were $7 \mathrm{E}+19 \mathrm{~cm}^{-3}$ of $\mathrm{Mg}$, the UID GaN had $1.0 \mathrm{E} 16 \mathrm{~cm}^{-3}$ of $\mathrm{Si}$ and $n-\mathrm{GaN}$ had $1.0 \mathrm{E} 18 \mathrm{~cm}^{-3}$ of $\mathrm{Si}$, as measured by secondary ion mass spectroscopy (SIMS). Samples suitable for transmission electron microscopy (TEM) were prepared using an FEI NOVA 200 dual-beam system, initially thinned at $30 \mathrm{keV}$ and then finally at 5 keV. A Philips-FEI CM-200 microscope operated at $200 \mathrm{keV}$ was mostly used for imaging.

Figures 1(a), 1(b) and 1(c) show schematics of the device structures for the Samples NE, E25 and N58, respectively, where the wavy lines signify that surface etching was carried out before initiation of the $p$ GaN growth. Figure 2(a) is a cross-sectional TEM image of Sample NE, which shows no visible sign of the presence of defects or precipitates. Figure 2(b) is a TEM image of Sample N25, and the presence of small precipitates in the $p-\mathrm{GaN}$ layer can be observed. Figure 2(c) is a TEM image of Sample N58. Precipitates are visible in the $p$-GaN material and the interface between $p$-GaN and UID layer is clearly visible. Figure 2(d) shows a higher-resolution image of the interface region. In the two etched samples, i.e. E25 and E58, no obvious etch damage is observed. Further investigations into the presence and nature of the precipitates in the $p-\mathrm{GaN}$ layers are ongoing [3].

\section{References:}

[1] K. Fujito et al., J. Cryst. Growth. 311 (2009), p. 3011-3014.

[2] Y. Zhang, A. Dadgar, T. Palacios, J. Phys. D. Appl. Phys. 51 (2018), p. 27.

[3] This work was supported by ARPA-E award DE-AR0000868. The authors acknowledge the use of facilities within the John M. Cowley Center for High Resolution Electron Microscopy at Arizona State University. 


(a) $500 \mathrm{~nm} p-\mathrm{GaN}$
UID-GaN $25 \mathrm{~nm}$
UID-GaN 9um
Sub.
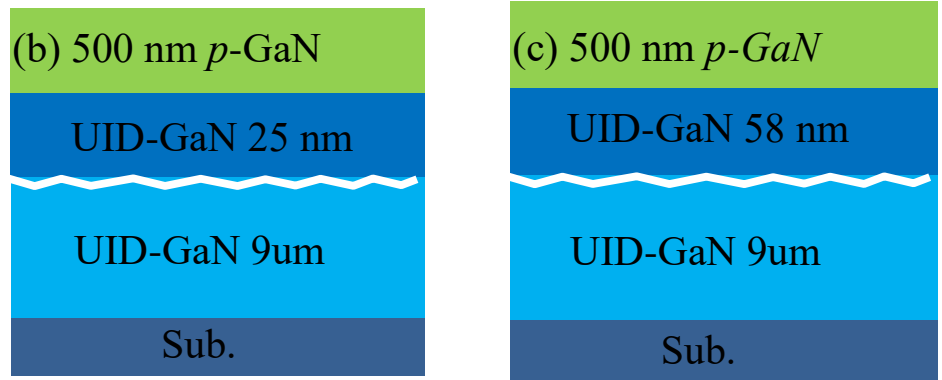

Figure 1. Schematics showing differences in nominal device structure: (a) NE; (b) E25; and (c) E58.

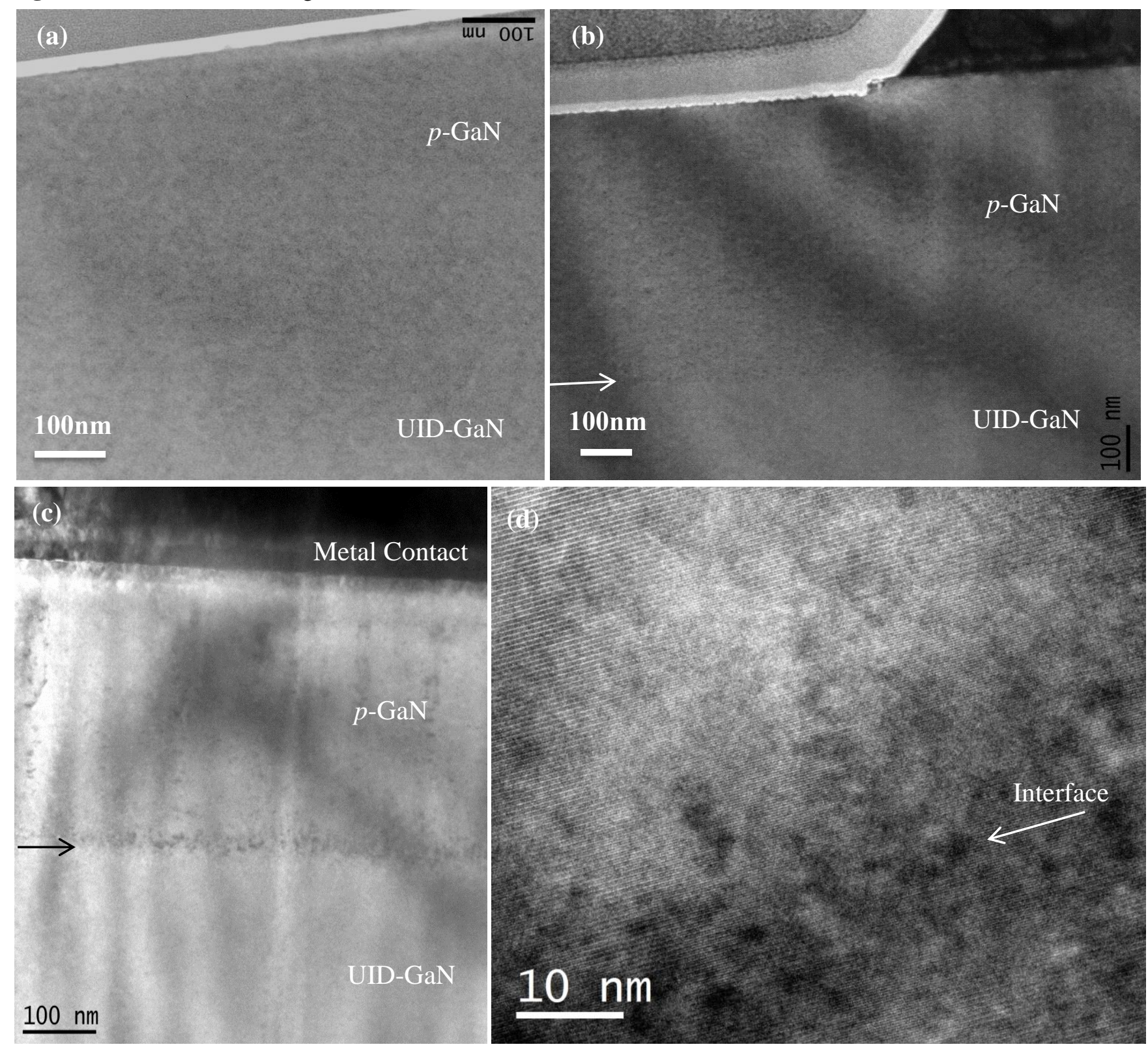

Figure 2. Cross-sectional TEM images of GaN-on-GaN Schottky diodes: (a) Sample NE showing no visible sign of GaN-GaN interface; (b) Sample E25 showing faint signs of small precipitates in $p$-GaN layer, (c) Sample E58 showing precipitates at GaN-GaN interface and also within the $p$-GaN layer, and (d) Higher magnification image showing interface region of Sample E58. 